\title{
Las acciones de transferencia de información y la comunicación
}

\author{
MARÍA NÉLIDA GONZÁLEZ DE GóMEZ \\ Investigadora y Profesora del Programa de Posgrado en \\ Ciencia de la Información, Universidad Federal de Río de \\ Janeiro, Escuela de Comunicación E-mail: nelida @ omega.Incc.br
}

\section{RESUMEN}

En este artículo se muestran algunos puntos de vista sobre la representación, transferenciay recuperación de lainformación. A partir de tales elementos, se afirma que es en las prácticas sociales, donde se construyen las evidencias informacionales, tanto las científicas como las de la vida cotidiana, por lo que la indización debe realizarse en el contexto de acciones contractuales y formalizadas de transferencia de información. Finalmente se menciona la teoría de las tres lecturas, a fin de observar los diferentes tipos de interpretación de significado que pueden darse al momento de analizar la información.

\section{ABSTRACT}

This paper shows some viewpoints on information representation, transfer and retrieval. From these elements it states that informational evidence, both scientific and every day, are constructed within the social practices.Thus, indexation must be undertaken within the context of contractual and formalized information transfer.

The three lectures theory is mentioned so as to observe the different types of interpretation of meaning that may arise when analyzing information.

\section{LOS PUNTOS DE PARTIDA}

A lgunas premisas, que servirán de punto de partida para nuestra argumentación, deben ser explicitadas:

1) La "Transferencia de Información", conforme nuestra concepción, abarca desde la producción del conocimiento y de la información hasta el uso de la información y su transformación en nuevos conocimientos.

2) La "Recuperación de la Información" dividida en dos etapas (primera, acceso a las referencias de los documentos y segunda, acceso a los documentos en sí) y en general, el sistema de información bibliográfica, es un segmento importantísimo de la "Transferencia de Información", pero no la cubre por entero.

3) Al mismo tiempo, consideramos la "Representación de la información" como un área clave para comprender la naturaleza y formas de intervención técnica y social en los procesos de comunicación científica y cultural. 
4) Los estudios de la "Representación de la Información" incluyen así, los específicos de la clasificación eindización de información documental, la representación de informaciones estadísticas en grandes bases de datos demográficos, los hipertextos, la representación de la información cultural en instituciones de memoria, como los museos y los archivos. Y estos estudios tratan de dilucidar las semejanzas y diferencias que estas representaciones tienen.

\section{SIG NIFICA DO, CONOCIMIENTO, INFORMACIÓN}

En ese horizonte de problemas, nos preguntaremos por la relación que existe entre información, significado, conocimiento y lenguajes documentales.

Los estudios de la información y de la documentación tienen hoy que ocuparse del significado en tres direcciones:

- Por la vía teóricay metodológica, deben atender a la actualización de las Ciencias de Interpretación del Texto.

- Por la vía práctica y factual, deben responder al impacto de las nuevas tecnologías intelectuales sobre el sistema de inscripción (los modos de fijar y reproducir significados). La oferta de conmutación digital casi ilimitada de los mensajes parece reunir en unaúnica red la ficción y la ciencia, la imagen, la palabra y el sonido, lo local y lo distante, el autor y el lector, el indizador, el editor, el empresario de la industria de la información.

- Por la vía ética y política, debe pensar en las condiciones de los procesos de identificación social y de la autonomía cultural frente a los nuevos mecanismos económicos y tecnológicos de metamodelización de las estructuras comunicacionales einformacionales, y de esta manera, nuestra propia experiencia individual y colectiva.

Por otro lado, hoy estarían sufriendo cambios profundos todas nuestras definiciones de valor gnoseológico.

Al mismo tiempo que parece abrirse indefinidamente el campo de las posibilidades informacionales, la red de la comunicación electrónica parece absorber anulando todas las formas previas de vinculación gnoseológica de la información.

El conocimiento, sin embargo, por su propia definición, parece haber necesitado, en su desarrollo, de esos espacios reguladores donde se construyen y se critican sus contenidos específicos.

De hecho, desde la formación de la "Sociedad Moderna", las disciplinas académicas y las especialidades, las unidades territoriales y sus fronteras geopolíticas, las instituciones de la cienciay de la tecnología, como las universidades e instituciones nacionales de investigación, actuaron como planos de integración epistemológicay social de los conocimientos, a los que aplicaban sus pautas de procedimiento y normativas. 
Así, conocimiento se aplicaría a un saber socialmente legitimado, que coloca una exigencia fundamentada de validez y especificidad, frente a todos los otros saberes, afirmando su potencia de aprehensión del objeto de su competencia, en el ámbito fenoménico y metodológico de su construcción. Y es esa tarea y exigencia de justificación lo que lo diferencia de otros saberes cuya vigencia y valor está incluida en el propio dominio de la actividad a la cual sirve, sin requerir actores, prácticas de construcción y cuerpos normativos específicos (así sucede con los saberes tradicionales sobre alimentación, nacimiento, salud, etcétera). En ese sentido, todo conocimiento es autorreferente: implica, desde el inicio, conocimiento y metaconocimiento.

Sin embargo, la posibilidad de relación entre los diferentes discursos científicos, parecería requerir de nuevo un tercer componente, trascendental respecto al plano de constitución de cada uno de los discursos singulares y segmentados y por eso ca paz de reunir los lenguajes-objetos en un nuevo plano de igualación y síntesis: una gramática natural, una lógica natural, un metalenguaje.

¿Encontraríamos ese plano en el nivel morfológico-sintáctico de la información digital o en el universo controlado de la comunicación documentaria organizada?

¿Es necesario un tercer componente, un instrumento de enlace, como condición de la comunicación de los conocimientos?

A partir de esta primera afirmación de cambios en los parámetros de definición del conocimiento y de la información, vamos a ocuparnos ahora de la representación del conocimiento a través de lenguajes documentales, en el contexto de acciones intencionales y formales de transferencia de información.

Entre las diferentes prácticas profesionales que tienen como objeto el análisisy la representación de la información, tenemos el de construcción de "modelos de información". En su versión más vigorosa, constituye lo que Lyntinenn denomina la visión del "mapeamiento de la realidad" y Eco, del "pensamiento fuerte".

El modelo legitimado por el "pensamiento fuerte", que es un pensamiento esencialmente modelador, tendría que satisfacer las siguientes condiciones:

1. Ser capaz de reducir la complejidad (en el dominio de lo modelado o de lo representado).

2. Ser heurístico; permitir que sean colocados en evidencia trazos del dominio del objeto que no eran perceptibles antes del modelo.

3. Ser homológico o isomórfico; remitiendo del mundo del modelo al mundo modelado (mundo real ).

4. Permitir predicciones, la anticipación del acontecimiento de determinadas ocurrencias o fenómenos.

El mayor problema del acto de modelar es entonces mantener al mismo tiempo dos reglas que se oponen: 
Por un lado, seleccionar atributos y relaciones propias del modelo, buscando la reducción de la complejidad del campo del objeto modelado. Por otro lado, mantener el isomorfismo modelo/ mundo o modelo/ objeto modelado: esto aseguraría la reversibilidad de la operación de reducción, y permitiría sustentar la capacidad predictiva del modelo.

Se trata, en cualquier caso, de un modelo construido por un observador externo al mundo/ objeto de la acción modeladora.

Siendo lo modelado del orden de la información, esto nos lleva a un nuevo problema: todo modelador de un objeto informacional y de acciones que tengan como objeto productos y servicios de información, tiene como objeto de modelaje una construcción informacional de otro actor (autor o agente de prácticas de información en el interior de acciones significativas). En ese sentido, un analista de información es siempre un metamodelador, cuyo modelo tiene como objeto las construcciones modeladoras de otros actores. En el mejor de los casos, un coproyectista y un coplanificador de acciones y objetos de información: un participante, junto a otros, del plano de metamodelización comunicacional de una experiencia colectiva. (Estarían juntos en ese plano de metamodelización, al menos, los cientistas, los educadores, los analistas de sistemas, los periodistas y comunicadores).

Esta situación de intervención en la comunicación de saberes culturales (conocimientos científicos más saberes y discursos estéticos, políticos, jurídicos) hace de la operación de modelar objetos de información una propuesta paradójica.

Se pretende, por un lado, que un actor (agente de la acción de transferencia de información), se mantenga como observador externo de los procesos cognitivosycomunicacionales de otros actores sociales que forman el universo de receptores 0 usuarios de la información (eintegran el mundo-objeto del modelo): es decir, supeditando procesos significativos a procedimientos observacionales y no comunicacionales de descripción y operacionalización. Por otro lado, se pretende intervenir en dominios específicos de producción de sentido, con la intención de su optimación, lo que solamente puede acontecer por la inclusión del actor agente de la transferencia y el receptor o actor final en una misma esfera de comunicación, como intralocutores.

Veamos qué sucede cuando entendemos la operación documental como una operación semántica. ${ }^{1}$

1 Kobashi, Informare v.2, n.2, 1996 G ardin, citado por Kobashi, asimila la operación de constituir representaciones a los procesos realizados en el interior de las ciencias que analizan e interpretan textos: el pasaje del texto original para ese género de "representaciones" sería una operación semántica, aunque sin obedecer a reglas precisas. 
La operación documental, como operación semántica, se realiza por medio del análisis del contenido textual del documento: los textos pasan por un conjunto de operaciones que lo transforman en un producto documental. ${ }^{2}$

Texto1

\section{Documento Primario}

Textos en lenguaje natural 0 en el lenguaje especializado del emisor

\section{Texto2}

\section{D ocumento secundario}

El producto de la operación

Operación semántica documental: las representaciones documentales

Existirían otras condiciones específicas del análisis documental, además de aquellas comunes a todo modelo: los principios y procedimientos usados en el AD deben ser rápidos y potentes, para dar cuenta de grandes volúmenes de textos y para dar acceso a la información actual.

Las principales operaciones, quevan a transformar un ítem de información en un producto documental de circulación en los espacios y procesos de la comunicación documental organizada, serían tres: ${ }^{3}$

a) lectura del texto;

b) selección del contenido informativo pertinente y relevante para un uso determinado;

c) representación de las informaciones seleccionadas para transformarlas en documentos manipulables, o documentos secundarios.

El análisis documental, en realidad, transforma el documento en tres productos principales de representación: dos de carácter temático y resultantes de la condensación del texto: el resumen y el índice; yuno, el propio registro documental normalizado, de carácter descriptivo y que remite a variables transtextuales (autor, edición, etcétera).

Los lenguajes documentales, en la operación documental como operación semántica, constituirían el "tercer componente" que permite ejecutar la operación de "traduc-

2 Kobashi, Informare v.2, n.2, 1996.

"A passagem do texto original para esse gênero de representação -empregaremos doravante este termo para designar o produto da análise documentária - é sem dúvida uma operação semántica, mesmo que ela não obedeça, na maioria das vezes, a nenhuma espécie de regra precisa, e que cada organismo de documentação, e mesmo cada analista, se limite a buscar no documento a ocorência de uma certa regularidade intema, fundada muito mais na experiência ou no hábito, do que em algúm tipo de procedimento explícito".

3 Idem El texto de K obashi nos ayuda a precisar los conceptos. El término representación sería un concepto preteórico, asociado por un lado a la descripción de aspectos que identifiquen materialmente los documentos y por otro, al proceso (indización, elaboración de resúmenes) y al producto (resúmenes, índices) de la condensación de los contenidos de textos. El acto documentario consistiría en la aplicación de metodologías que permitan construir objetos que materializarán la comunicación documental. Comunicación documental es un proceso que envuelve la codificación y descodificación de contenidos informacionales, o sea, de tratamiento y recuperación de la información, teniendo como producto la representación. 
ción" del texto original al texto condensado, con el objetivo de alcanzar una circulación reguladora y normar la información en su esfera de destino. Los lenguajes documentales son lenguajes controlados y más o menos formalizados, para lo cual tienen querestringir aprioriel campo de aplicación y uso delos significados indizados.

Serían éstos los presupuestos de la operación documental:

1) Es posible identificar la información principal del texto.

2) Existe un significado fijo, un vocabulario privilegiado o una interpretación privilegiada de un texto, que expresa esa "información principal".

3) Existe un principio de isomorfismo o de equivalencia ${ }^{4}$ entre el producto de la operación documental ( palabras claves; descriptores; resúmenes; bibliografías; bases de datos referenciales; guías de fuentes) y el objeto de la operación documental (texto, documento), de manera que la presencia de uno, permite predecir el valor informativo o semántico del otro.

4) Es posible anular los valores expresivos de un texto; la representación sería "neutra" en relación con los valores expresivos.

5) Es posible diferenciar variables intemas de variables extemas a la operación documental, de modo que la operación se mantenga en un ámbito bien demarcado y controlable de variables internas y semánticas (como las variaciones del grado de precoordinación de los términos). Q uedarían fuera de esterecorte semántico de la operación documental, como variables externas: a) las variables descriptivas de un ítem de información ( consideradas categorías de datos de catalogación); b) las variables que describen los agentes de tratamiento de la información y sus prácticas profesionales (analistas de información y variaciones de competencia y desempeño); c) las variables contextuales y organizacionales (como normasy patrones de excelenciay políticas de información).

Esa equiparación del análisis documental y los procedimientos de lecturay análisis textual, que mantiene su validez como elección metodológica, en una estrategia definida de investigación, tiene o tra premisa implícita: el predominio de lo dicho sobre el decir.

Veremos algunas consecuencias de esta estrategia, para después proponer una estrategia alternativa. Podemos afirmar que en esta definición de la operación documental, el analista de información estaría forzado a buscar el equilibrio entre dos posibilidades opuestas: la de una lectura imperfecta o la de una superinterpretación y de una metaescritura, de una autoría de segundo grado.

La imperfección de la lectura resultaría de la posición del indizador frente al texto y su relación con el "tercer componente" de la traducción.

4 Idem "Embora a informação documentaria seja obtida, de um lado, pela neutralização do poder expressivo do texto e, de outro, moldada de acordo com regras previamente determinadas, prevalece a ideia de algo que, a pesar de ser originalmente diferente do original, portanto "representaçào", é equivalente a ele, do ponto de vista do conteúdo informacional." 
Primero, la imperfección de la lectura resultaría de la posición intermedia frente al texto, fuera del polo del autor del texto y del polo del lector del texto, lo queagrega a la indeterminación del texto, la polifonía de las interpretaciones que mediatizan su lectura.

Segundo, la utilización del lenguaje documental, el tercer componente o instrumento conector, realiza su operación como un paralenguaje 0 un metalenguaje, construido por procedimientos selectivos sobre las selecciones de significados realizadas en el momento de la inscripción textual, y conforme una estrategia de selección que privilegia el punto devista del sistema o del usuario final dela información.

Los tesauros son repertorios o listas de términos autorizados, constituidos por unidades-descriptoresy no-descriptores, pertenecientes a un dominio particular del conocimiento, relacionados semántica y lógicamente. Son utilizados para caracterizar tanto el contenido de un documento como el contenido de las preguntas propuestas por el usuario. Su función es por eso, la de servir como intermediario entre los documentos y los usuarios.

Los elementos de los lenguajes especializados son los términos. Mientras los términos son obligatoriamente extraídos del discurso, los descriptores son unidades preferencialmente conocidas por los usuarios:

D e una manera más precisa, sucede que el descriptor no representa de hecho el conocimiento, o no lo hace integralmente, ya que no es una unidad monorreferencial - como lo es el término- pero sí, preferencial.

D e eso resulta que el descriptor no tiene la precisión del término. ${ }^{5}$

Los descriptores, elementos del lenguaje de indización, tendrían que ser portadores de una información tal que permitiera relacionar el documento con un objeto dela realidad extralingüística. Mas son palabras indeterminadas en cuanto a su valor de referencia. Las palabras tienen propiedades: sólo el discurso remite o construye universos de referencia. El paso de la palabra al discurso equivaleal paso de la lógica intencional a la lógica extensional (lo que implicaría la utilización de predicados complejos o relacionados) ${ }^{6}$

En el otro extremo, dentro del tratamiento sistémico, se presupone un centro de decisión que tiene el poder y los medios de elegir un lenguaje legítimo del sistema, de modo que el analista de información se convierte en un intérprete privilegiado, cuya lectura encierra el potencial de sentido de un texto en una única interpretación, de la cual depende su presencia y ausencia en los ciclos de la comunicación documental organizada. En realidad, él se convierte en un megautor, que transforma la diversidad de su base de documentos en un nuevo y único texto (el periódico de resúmenes, la bibliografía, la base de datos bibliográficos). Como el filólogo, que fija

5 H. Kuramoto. "Uma abordagem alternativa para o tratamento e a recuperação de informação textual: os sintagmas nominais". Ci.Inf, v.5, n.2, p.182-192, 1996.

6 R. Fugmann. "Na interactive classaurus on the PC". Intemational ClassificationV.17, n.3/4, p.133-137, 1990. 
el significado del texto interpretado en un metanivel, el analista de información interpreta un texto con un texto.

\section{EL TEXTO, LA LECTURA, LA INTER PRETACIÓN : LAS FRONTERAS DEL ANÁLISIS DE INFORMACIÓN}

Podemos definirun texto como un discurso elaborado con un propósito deliberado. Incluimos en esta categoría, además de los textos del lenguaje verbal, diagramas, mapas, mensajes fílmicos o iconográficos.

A la indeterminación del significado textual, Levvy agrega la característica de ser una unidad virtual. Si la lectura es su actualización, la lectura sería siempre una manera nueva y singular de resolver el problema del sentido. ${ }^{7}$

El hipertexto solamente exhibiría algunas de las propiedades que siempre estuvieron en el texto y en la lectura.

La lectura tanto bucea en el interior del texto, sus remolinos y vacíos, como también lo ignora, lo atropella, lo atraviesa por las márgenes persiguiendo indicios del autor, del traductor, del editor, del tipógrafo. Sobrela linealidad de la escritura, buscamos las entrelíneas, los desvíos que el propio autor parece ignorar. Leer es construir y una construcción que nunca alcanza una forma final.

Pero si el sentido nunca está completo en el texto, ¿¿sobre qué trabaja el análisis documental? ¿Cómo podríamos identificar el "sobre qué" de un texto, aquello que para Hjorland ${ }^{8}$ estaría definido por el conjunto de las posibilidades epistemológicas de realizar el valor informativo-semántico de un texto?

La virtualidad dela obra cultural no sólo permite que diferentes sujetos hagan diferentes lecturas de un texto, sino también que un mismo sujeto construya diferentes significados en las diferentes lecturas de un mismo texto.

Es preciso avanzar en la búsqueda de recursos conceptuales.

Si el sentido no reside exclusivamente en el texto, tampoco es exclusivo del autor 0 del lector. A diferencia del concepto de interpretación (centrada en el receptor) 0 de la concepción clásica del texto (centrada en el emisor), el concepto de negociación de significados (transadion) implica la no direccionalidad de la construcción de sentido y la posibilidad de interacción y de "intralocución".

Para G eertz, ${ }^{9}$ es necesario pasar de lainscripción como producto al acto de inscripción, al proceso de fijación del sentido. ¿Cómo se conduce la inscripción de la acción? ¿por qué medios? ¿cómo es elaborada? ¿qué significados se retienen y fijan el flujo de los acontecimientos? es decir ¿qué de lo que sucede se convierte en his-

7 P. Levvy. Queéovirtual? RJ, Ed.34, 1996. p.35.

8 B. Hjorland. "The concept of "subject" in Information Science". Jarmal of Doumentation V.48, n.2, p.172-200, 1992.

9 G eertz. Local Knoweekge Further EssaysinintepretativeAnthropodogy. Harper Collins, 1983. 
toria? ¿qué del pensar se transforma en pensamiento? ¿qué de la acción cultural se transforma en obra? en fin, ¿qué del documentar se convierte en documento?

Laproducción de sentido, a partir de cualquier sistema simbólico (repertorio técnico, pintura impresionista, programa de computador) es siempre organizada por reglas construidas y negociadas en el contexto de la experiencia colectiva.

Las reglas por las cuales un conjunto simbólico tiene sentido y sin las cuales ningún conjunto de símbolos tendría sentido ${ }^{10}$ definen y son definidas por comunidades de interlocución y representación. Las mismas actúan como restricciones sobre la autonomía semántica de los individuos, asociando lo privado a lo público y sirviendo de instrumento a las formas simbólicas del poder.

La reglas organizan, así, las experiencias práctico-discursivas, generando regularidades a partir del contrato local de los participantes de una red de relaciones sociales mediatizada por el lenguaje. D eben tener un carácter público y no privado.

Laproducción social de conocimientos científicos perteneceal campo de las posibilidades práctico-discursivas. Podríamos afirmar, luego, que el modo de vinculación de los conocimientos responde a la forma de reglas y no a estructuras apriorísticas y universales. En ese sentido, G eertz considera las prácticas científicas como "formas de vida".

Esa "garantía colectiva" (commmal warrants) de la interpretación no intervendría sólo en la construcción de significados denotativos comunes, sino también en la construcción de macroestructuras que facilitarían el control social de grandes grupos o áreas de la actividad humana.

Las reglas no son, a pesar de eso, fijas: una de las mayores funciones de la crítica sería la renegociación de las reglas de interpretación.

Pensamos quela acción de transferencia de información interviene en la misma dirección: ya sea como una renegociación de significados, ya sea por la construcción de estructuras de información. ${ }^{11}$

\section{LA TEORÍA DE LAS TRES LECTURAS}

10 Ibidem

11 M. N. G omez, ReatóiodePesquisa: "La representación instrumental procede a fijar, en la dinámica de la transmisión de significados, estructuras de información. Éstas intervienen como operadores semánticos, con la función de agregar y disociar informaciones de modo significativo para permitir su busca segmentaday su recuperación selectiva. D enominamos estructuras de información a los procedimientos y sus resultados, orientados al control de la transmisión de significados, teniendo como objetivo aumentar la previsibilidad de una interpretación preferencial, en un contexto específico de comunicación de la información. Las estructuras de información serían configuraciones estables de significados, constituidas por categorías y redes conceptuales, palabras y agregados de palabras o por otros elementos de representación (icónicos, por ejemplo)." 
Como corolario del principio de la indeterminación del sentido del texto, las nuevas teorías de la lectura ${ }^{12}$ afirman que lo que hace que un texto sea científico 0 no científico no es el propio texto, sino la forma de leerlo. Las diferencias de sentido se establecen en la relación entre texto y lector. Así, la inestabilidad del significado de un texto no es solamente propia de la literatura de ficción, sino de cualquier escritura.

Podemos pensar entonces en una lectura estética, que sería aquella en que el lector está centrado en lo que vive en cuanto lee, en sus propias vivencias y en una lectura informacional, que sería aquella en que el lector está centrado en la información que obtiene del texto. Sería el tipo de lectura de la argumentación, una lecturano accidental, sino que tiene el propósito de actualizar la base de conocimientos del lector o llenar una laguna.

A esas dos formas de lecturas, proponemos agregar otra, unalectura del analista de información, ni imperfecta ni metalectura, sino otra lectura: la lectura de la metainformación.

La lectura proposicional del analista de información se dirige a la metainformación, y las condiciones so bre las cuales es capaz de relacionar la información con la información, permitiendo la realización del valor semántico de la información.

El propósito de estalectura es la transferencia de la información, para que ésta rea lice su máximo valor semántico o cognitivo en un lector final, el usuario.

En ese sentido, el analista de información antes que un intérprete, es un re-negociador de los sentidos negociados entre el autor y el lector.

Esa lectura metainformacional tiene como objeto la relación de las informaciones entre sí yel modo como estas relaciones llevan a la articulación entrelainformación y el conocimiento. Produce así un nuevo tipo de sentido, que podemos llamar metaconocimiento.

La información y la metainformación poseen un valor en la construcción social del conocimiento y del metaconocimiento, pero ese valor no es el mismo que el valor de verdad, conjetura o falsedad que se puede atribuir a un conocimiento.

La pregunta por ese valor sería: ¿cuáles son las condiciones metainformacionales de un testimonio o evidencia informacional? Y de nuevo nos preguntaríamos si se trata de las condiciones de definición de una "evidencia" que podrían ser establecidas aprioi, las mismas en todos los casos, o si se trata de una construcción a posteion, de modo que esas condiciones metainformacionales de una evidencia informacional podrían variar, caso a caso.

Nuestra hipótesis es que la metainformación tiene el carácter de reglas producidas por las personas, sus prácticas y sus cuadros de referencia institucionales.

12 Brent D ough. Reedingas Rhetrical Invention: Knowkedge, Pessuasion and theTeading of Reserch-Based Writing Urbana, National Council of Teachers of English, 1992. 
Tenemos también algunas nociones de posibles criterios de valor que definirían calidades de información: lo verosímil, lo auténtico.

D avid Bearman, ${ }^{13}$ justamente, se refiere a la "crisis de la evidencia" y de la accountabilityen la sociedad de la información: los registros electrónicos, los transientmessa ges dejan preguntas no respondidas claramente todavía, sobre su acceso futuro, sobre las condiciones de su durabilidad, sobre las marcas de su identificación.

Las Ciencias Humanas, por ejemplo, dependen de la posibilidad de estudiar textos, filmes, imágenes originales, de poder hacer atribuciones correctas y de tener certeza de la autenticidad de un vestigio cultural. Si no conocemos el contexto en que una información fue creaday quiénes participaron en esa creación, muchas de las cuestiones que le serán presentadas a la información quedarán sin respuesta (por más abundante y variada que ella sea).

¿Cómo el futuro podrá conocer nuestra época?

Bearman coloca cuatro problemas respecto a la información archivística, que es una de las fuentes del conocimiento jurídico e histórico, pero también del conocimiento económico y de los negocios: la preservación de señales o 'bits'; la "preservación intelectual"; la preservación "funcional" o la garantía de independencia del software y de la posibilidad de migración de un medio a otro medio; el establecimiento de nuevos patrones sociales y legales de "evidencia".

La inestabilidad del registro electrónico, la comercialización de un gran número dediferentes medios de almacenajey de diferentes formatos de registros, todo parece indicar una rápida obsolescencia o pérdida del registro electrónico. Existen medios de lectura y copia que permiten el transporte o la migración de los datos de un software a otro, de un hardware a otro: pero ¿cómo saber cuál es el grado de pérdida en esa transposición de un medio a otro ? ¿cuál el residuo de significado sin traducción tecnológica?

En el plano de lainformación digital ¿cómo podemos establecer cuando tenemos un "documento" o un conjunto de datos registrados?

Gracias ala escritura, la unidad de registro parecía ganar una relativaindependencia de sus contextos de producción. El texto digitalizado, sin embargo, es parte dependiente de un sistema de inscripción, almacenaje y transmisión, y sólo dentro de ese sistema mantiene su potencia funcional. Un hipertexto en Internet posee relaciones y posibilidades de intertextualidad que sólo se mantienen mientras está en la red: un conjunto de copias de textos en papel nunca será equivalente al hipertexto y sus potencialidades funcionales. Las relaciones entre sites u objetos informacionales son complejas y sin reproducción adecuada fuera del ambiente tecnológico de su funcionamiento.

13 D. Bearman. Virtual Archives. Http/ / unwodcarg5046/ weibd/ ardtf/ beammantxt. 17/ 05/ 97. 
Entre losimpactos de la disolución de los atributos testimoniales del registro, tenemos también la pérdida de la unidad narrativa, de la unidad de acción, de tiempo y de lugar de la narrativa clásica. El montaje singular del lector individual trastorna la dspositiode la vieja retórica.

En el medio digital, la lectura se aproxima a la edición y a la escritura; se forman colectivos de lecturas y lectores que establecen un continumque iría de la lectura de un sujeto individual en un único texto, a las redes textualizadas donde muchos sujetos simultáneamente conectan textos, los anotan, los aumentan con sus propias inscripciones. Cada uno no sólo aumenta el repositorio de informaciones: agrega al conjunto un wandkingpaint of view un punto de vista desde una posición distante sobre el conjunto, injerta en la red una nueva malla de singularización.

\section{EL POSTULADO DEL CONTRATO COMUNICACIONAL}

Todo nos lleva a considerar que debemos analizar las prácticas de información a la luz de una sociedad posconvencional, resultante de la metamodelización intencional eintensiva dela comunicación y dela información (con la intervención de los medios de comunicación social, la telemática y las industrias de la cultura y de la información, así como de bibliotecas, centros de documentación y archivos).

Las prácticas y acciones de información formarían parte de un continuum que tiene en uno de los extremos la comunicación, como forma ideal de construcción intersubjetiva de las informaciones, en un marco de plena reciprocidad normativa y argumentativa, y en otro de los extremos la transferencia de información, como situación de total asimetría entre los sujetos emisores y receptores participantes de esa acción. Suponemos que nunca nos encontraremos frente a una situación de pura comunicación, pero que también es imposible la absoluta pasividad de la recepción en una situación de transferencia totalmente asimétrica. La transferencia de un minimunde información sobre el cual se sustentaría la socialización moderna, requiere un minimnlógico y ético de normas, conocimientos y significados recíprocamente compartidos.

Frente a las presuposiciones radicales de conmensurabilidad o inconmensurabilidad de las teorías y de los discursos sociales, contraponemos la historicidad de una situación donde la traducción y los movimientos circulares de la producción cultural del sentido, se enfrentan con los hiatos de la comunicación que resultan de la exclusión, el secreto, los privilegios, la desinformación.

Reuniendo en este punto nuestras anteriores afirmaciones, diremos que la definición de una evidencia informacional solamente sucede conforme a reglas, por medio de relaciones contractuales, implícitas o explícitas, en contextos institucionales específicos.

Agregamos ahora que esas reglas tendrían que satisfacer las expectativas de un acto de argumentación, como articulación no ética del leer y del escribir, del hablary del escuchar, del informar, el informarse y el ser informado. 
Es en el espacio virtual de la argumentación que todas las demandas de evidencias son problematizadas y sujetas a las condiciones de la aceptación.

En ese cuadro, el contrato comunicacional sería condición y premisa de la posible acción colectiva y presupuesto de toda formulación del contrato social.

Sería al menos, y sujeto a su validación en esferas de argumentación, una posible definición del conocimiento científico en los nuevos contextos de su producción y comunicación.

El analista de información encontraría en ese contrato comunicacional los parámetros de la renegociación del sentido.

Para Wersigit la ciencia de la información tendría como objeto estudiar acciones de información que tienen como finalidad la provisión de fuentes externas de argumentación.

La participación en esferas contractuales y argumentativas exigirá que los analistas y otros profesionales de la información coloquen públicamente las reglas de los juegos de la información, y permitan que los otros participantes de ese juego discutan esas reglas y produzcan otras. Los juegos participativos de información son al menos una condición de la futura "Ciudad de la Información”, más democrática y equitativa.

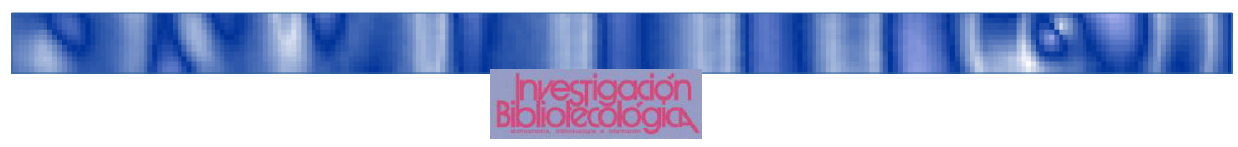

14 G. Windel, G. Wersit, "Information Science needs a Theory of 'Information Actions"”.

Social ScienceInfomation Studies, n.5, p.11-23, 1985. 